\title{
Expression of the serine protease inhibitor serpin A3 in human colorectal adenocarcinomas
}

\author{
JAN DIMBERG ${ }^{1}$, KARIN STRÖM $^{2}$, STURE LÖFGREN $^{2}$, NIKLAS ZAR $^{3}$, \\ ANDERS HUGANDER ${ }^{3}$ and ANDREAS MATUSSEK ${ }^{4}$ \\ ${ }^{1}$ Department of Natural Science and Biomedicine, University College of Health Sciences; \\ Departments of ${ }^{2}$ Clinical Microbiology, ${ }^{3}$ Surgery and ${ }^{4}$ Laboratory Medicine, \\ Ryhov County Hospital, SE-551 11 Jönköping, Sweden
}

Received December 15, 2010; Accepted January 18, 2011

DOI: $10.3892 / \mathrm{ol} .2011 .280$

\begin{abstract}
Proteases facilitate a number of steps in cancer progression. The serine protease inhibitors (serpins) are a protein superfamily with inhibitory activity against proteases. One of these proteases, serpinA3, appears to have a multifaceted role and is associated with inflammatory reactions, Alzheimer's disease, malignant melanoma and gastric cancer. To gain insight into the potential effect of serpinA3 on colorectal cancer (CRC) we determined whether serpinA3 is altered in colorectal tissue or plasma in CRC patients. Collectively, by using ELISA we noted a significantly lower serpinA3 level in cancer tissue compared to paired normal tissue. Moreover, the tumour serpinA3 level tended to be higher in disseminated disease as compared to localised disease. No significant difference in the plasma levels of serpinA3 was noted in the patients when compared to the controls. However, plasma serpinA3 and C-reactive protein (marker of inflammation) in the CRC patients and controls were significantly positively correlated. To confirm and detect localization of serpinA3 expression, immunohistochemistry was performed. Immunohistochemistry showed heterogeneous immunoreactivity in epithelial cells in the cancer and normal tissue and extracellular staining within bands of stroma as well as in some stromal cells. A Taq Man system was used to investigate a single nucleotide polymorphism (rs4934) in the serpinA3 signal sequence gene with supposed effect on serpinA3 secretion and expression. No significant difference was observed between CRC and control subjects regarding genotype and allelic distributions, nor were associations noted between clinical characteristics and serpinA3 levels. In conclusion, an altered serpinA3 concentration in CRC tissue may be a potential biomarker in CRC progression. SerpinA3
\end{abstract}

Correspondence to: Dr Jan Dimberg, Department of Natural Science and Biomedicine, University College of Health Sciences, Box 1026, SE-551 11 Jönköping, Sweden

E-mail: jan.dimberg@hhj.hj.se

Key words: serpinA3, polymorphism, colorectal cancer concentrations in plasma appear to be correlated with systemic inflammation, but do not appear to be specific to CRC patients. Further studies are warranted to improve our understanding of the role of serpinA3 in CRC.

\section{Introduction}

Studies have indicated that proteases have fundamental roles in pathological processes in cancer by degradation of basal membranes and extracellular matrix (1). Matrix metalloproteinase (MMP) expression has been linked to tumour aggressiveness and tumour invasion in a wide range of cancers, such as colorectal cancer (CRC) (2-4). Cathepsins, another class of proteases, also play a role in invasion and metastasis of CRC (5). Serine protease inhibitors (serpins) belong to a protein superfamily with inhibitory activity against proteases. These serpins are involved in various physiological processes and in the regulation of events associated with inflammatory reactions and connective tissue turnover $(6,7)$.

SerpinA3, also referred to as $\alpha$-1-antichymotrypsin, is a member of the serpin family (6) and is an acute phase protein which increases in the blood during the inflammatory process $(8,9)$. The inhibitory effect of serpinA3 appears to be more specific against neutrophil cathepsin G, mast cell chymases and pancreatic chymotrypsin $(8,9)$.

SerpinA3 is synthesised primarily by hepatocytes, bronchial epithelial cells and monocytes but is also expressed in a variety of organs such as kidney, brain and prostate $(8,9)$. Overexpression of proteinase inhibitors including serpinA3 was found to result in poor prognosis and malignancy in gastric cancer (10).

The serpinA3 gene is located on chromosome 14 in humans, and a signal peptide polymorphism at codon 17 (A/T) in the serpinA3 gene has been associated with susceptibility to Alzheimer's disease and may affect age-at-onset and disease duration (11). A single nucleotide polymorphism (SNP) (rs4934) is located in the signal peptide region (at codon 15) in exon 2. It has been postulated that this genotype affects the expression of serpinA3 protein (12-14). Moreover, studies have proven that this genetic variant is a risk factor for aneurysmal subarachnoid hemorrhage in a Polish (12), but not in a Chinese (13) or Japanese (14) population. 
Data concerning the expression profile of serpinA3 in human CRC are limited. Therefore, the protein expression of serpinA3 in CRC tissue and plasma from CRC patients and its association with clinical parameters were determined. Screening for the serpinA3 gene polymorphism (rs4934) was carried out to evaluate a possible correlation with clinical outcome of CRC. Since proteases can be associated with the tumour cells themselves (1), an in vitro invasion assay was used to investigate the effect of exogenous serpinA3 on CRC cell (Caco2 and HT-29) invasiveness. Moreover, levels of C-reactive protein (CRP) were investigated to assess the effect of systemic inflammation (15) on the level of serpinA3.

\section{Materials and methods}

Patients and controls. This study comprised blood samples from 311 consecutive CRC patients from southeastern Sweden. The samples were collected from patients who underwent surgical resections for primary colorectal adenocarcinomas at the Department of Surgery, Ryhov County Hospital, Jönköping, Sweden. Clinicopathological characteristics from the patients were obtained from surgical and pathological records. The patient group comprised 166 males and 145 females with a mean age of 70 years (range 29-93). The tumours were localised in the colon $(n=162)$ and rectum $(n=149)$ and were classified according to Dukes' classification system: stage A $(n=53)$, stage $B(n=128)$, stage $C(n=107)$ and stage $D(n=23)$. Blood donors $(n=359)$, from Ryhov County Hospital, with no known CRC history were from the same geographical region as the CRC patients and were selected as controls. This control group consisted of 207 males and 152 females with a mean age of 67 years (range 50-80).

Blood samples were centrifuged to separate plasma and blood cells and both were stored at $-70^{\circ} \mathrm{C}$.

Tissue samples and lysates. This study utilised tissue samples which were available from 104 of the 311 CRC patients. The tumours and matched normal tissue from 61 males and 43 females with a mean age of 68 years (range 29-83) were used. The tumours were classified according to Dukes' classification system: stage A ( $n=17)$, stage $B(n=45)$, stage C $(n=33)$ and stage $D(n=9)$. The tumours were localised in the colon $(n=61)$ and rectum $(n=43)$. Tumour tissue and adjacent normal mucosa $(\sim 5 \mathrm{~cm}$ from the tumour) were excised from each patient and immediately frozen at $-70^{\circ} \mathrm{C}$ until analysis.

Frozen tumour tissue and normal mucosa were thawed, homogenised in ice cold lysis buffer containing PBS $(9.1 \mathrm{mM}$ dibasic sodium phosphate, $1.7 \mathrm{mM}$ monobasic sodium phosphate, $150 \mathrm{mM} \mathrm{NaCl}, \mathrm{pH} 7.4$ ) and $1 \%$ Nonidet P-40, $0.5 \%$ sodium deoxycholate, $0.1 \%$ sodium dodecyl sulphate (SDS), $100 \mu \mathrm{g} / \mathrm{ml}$ phenylmethylsulphonyl flouride (PMSF), $2 \mu \mathrm{g} / \mathrm{ml}$ aprotinin, $1 \mathrm{mM}$ sodium orthovanadate and $1 \mu \mathrm{g} / \mathrm{ml}$ leupeptin. The lysate was placed on ice for $30 \mathrm{~min}$ and then centrifuged at $13,000 \mathrm{x} g$ for $10 \mathrm{~min}$. Protein content of the supernatant fluid was determined for each sample using the Bradford protein assay (Bio-Rad Laboratories, UK).

Plasma samples. A total of 133 of CRC patients and 122 of the controls were available for plasma collection. The CRC patient group comprised 75 males and 58 females with a mean age of 68 years (range 29-89). The patient tumours were classified according to Dukes' classification system: stage A $(n=21)$, stage $B(n=57)$, stage $C(n=45)$ and stage $D(n=10)$. A total of 61 tumours were located in the rectum and 72 in the colon. Controls consisted of plasma from 62 males and 60 females with a mean age of 63 years (range 56-68).

Assay for serpinA3 and $C$-reactive protein concentrations. SerpinA3 was measured in tissue and plasma using a commercially available enzyme-linked immunosorbent assay (ELISA) kit (Immunology Consultants Laboratory, Inc., USA) and performed according to the manufacturer's instructions. The tissue levels of serpinA3 from the tumour and paired normal tissue were expressed as nanograms per milligram (ng/mg) of protein. CRP in plasma was measured using an immunoturbidimetric method (ADVIA 1800 Chemistry System, Siemens, USA). The plasma serpinA3 and CRP from CRC patients and controls were expressed as micrograms per millilitre $(\mu \mathrm{g} / \mathrm{ml})$.

Immunohistochemistry. Eleven tumour samples were obtained for immunohistochemical staining. To study the cell type origin of serpinA3 expression, staining was performed using a standard protocol on $4-\mu \mathrm{m}$ sections from formalin-fixed paraffin-embedded tissue blocks. Antigen retrievel was performed by microwave treatment in citrate buffer ( $\mathrm{pH}$ 6.0). Endogenous peroxidase activity was quenched by treatment with $3 \%$ hydrogen peroxide for $5 \mathrm{~min}$. Sections were subsequently incubated with a primary goat anti-human serpinA3 antibody (R\&D Systems, Minneapolis, MN, USA). After rinsing in Tris-buffered saline, sections were incubated with secondary biotinylated horse anti-goat antibody (Vector Laboratories, Burlingame, CA, USA). Avidin-biotin peroxidase complexes (Dako Cytomation, Denmark) were added followed by visualization with 3,3'-diaminobenzidine tetrahydrochloride (Dako Cytomation). Sections were counterstained with Mayer's hematoxylin (Histolab Products, Sweden). As negative controls, the primary antibodies were replaced by an isotype control IgG. Staining procedures were performed according to the manufacturer's instructions.

DNA extraction and genotype determination. DNA was isolated from blood from the patients and blood donors using the QiaAmp DNA blood kit (Qiagen, CA, USA). DNA samples were genotyped using the 5'-exonuclease allelic discrimination assay (Applied Biosystems, CA, USA). The Taq Man SNP genotyping assay was used for analysis of the serpin A3 genotype rs4934 (Applied Biosystems). DNA (10 ng) was amplified in a total volume of $12 \mu \mathrm{l}$ containing Taq Man Universal PCR Master mix (Applied Biosystems), included in the SNP genotyping assay. Amplification was performed using an initial cycle at $50^{\circ} \mathrm{C}$ for $2 \mathrm{~min}$ followed by 1 cycle at $95^{\circ} \mathrm{C}$ for $10 \mathrm{~min}$ and finally 40 cycles at $95^{\circ} \mathrm{C}$ for $15 \mathrm{sec}$, then $60^{\circ} \mathrm{C}$ for $1 \mathrm{~min}$. A post PCR endpoint reading was performed on each plate using the 7500 Fast Real-Time PCR system (Applied Biosystems). The manual calling option in the allelic discrimination application ABI PRISM 7500 SDS software version 1.3.1 was then used to assign genotypes.

Cell lines and invasion assay. Two established human colon cancer cell lines Caco-2 and HT-29 were purchased from 
American Type Culture Collection (ATCC, Rockville, MD, USA). The cell lines were grown according to the supplier's instructions and the growth media were Eagle's minimum essential medium (Caco-2) and McCoy's 5a (HT-29).

The cell invasion assay was carried out with 24-well (lower chambers) and 12 transwell chambers (inserts) (BD BioCoat Matrigel Invasion Chamber; BD Biosciences, Bedford, MA, USA) following the manufacturer's instructions. Briefly, for each type of CRC cell line, a cell suspension $\left(5 \times 10^{4}\right.$ cells $\left./ \mathrm{ml}\right)$ in serum-free media in the absence or presence of full-length protein serpinA3 (Immunology Consultants Laboratory) was used. The concentrations of serpinA 3 in the cell suspensions were $0,10,100$ and $2000 \mathrm{ng} / \mathrm{ml}$. The inserts were placed in the lower chambers containing fetal bovine serum media serving as a chemoattractant. Each insert contained an 8-micron pore size PET membrane with a thin layer of Matrigel matrix serving as a basement membrane and an invasion barrier. The cells that invaded the basement membrane following incubation at $37^{\circ} \mathrm{C}$ in $5 \% \mathrm{CO}_{2}$ for 22 and $44 \mathrm{~h}$ were fixed, stained with hematoxylin and eosin and counted in 10 random microscopic (x200) fields. Experiments using Caco-2 were performed twice and HT-29 four times in triplicates.

Statistical analysis. Differences in the frequencies of the serpinA3 gene polymorphism between CRC patients and the control group and between clinical data within the CRC subgroup were analyzed using the Chi-square test. HardyWeinberg equilibrium was tested for the genotypes. Differences in serpinA3 levels between the tumour and paired normal tissues were examined using the Wilcoxon's signed-rank test. Differences in serpinA3 and CRP levels between unpaired groups were tested using the Mann-Whitney $\mathrm{U}$ test, and correlation coefficients were determined using the Spearman's rank correlation test. Statistical analyses were performed using SPSS for Windows computer package, 2005 (Rel. 14.0, SPSS Inc., Chicago, IL, USA). Results were considered significant at $\mathrm{P}<0.05$.

\section{Results}

Protein levels of serpinA3 in colorectal tissue. To investigate the potential role of serpin $\mathrm{A} 3$ as a tumour marker the concentration of this protein in CRC and paired normal tissue from 104 CRC patients was measured using ELISA. The levels of serpinA3 in the tumour tissue (median $205 \mathrm{ng} / \mathrm{mg}$; range 18-1082) showed a significantly lower $(\mathrm{P}<0.001)$ concentration in comparison with the normal tissue (median $270 \mathrm{ng} / \mathrm{mg}$; range 39-956) (Fig. 1). Assessment of the relative expression (tumour vs. normal tissue) showed that in $77 \%$ of the cases the expression was down-regulated. Furthermore, the protein levels of serpinA 3 in the tumour and normal tissue were found to be significantly positively correlated $(r=0.50, \mathrm{P}<0.001)$ (data not shown).

Following subdivision of the patients according to localised (Dukes' A+B) and disseminated (Dukes' $\mathrm{C}+\mathrm{D})$ disease, the tumour level of serpinA3 in Dukes' $\mathrm{C}+\mathrm{D}$ disease (median $227 \mathrm{ng} / \mathrm{mg}$; range 22-1082) was higher compared to the level in Dukes' A+B disease (median $170 \mathrm{ng} / \mathrm{mg}$; range 18-881) $(\mathrm{P}=0.057)$ (Fig. 1). The tissue serpinA3 protein level in localised disease was not significantly different $(\mathrm{P}=0.669)$ compared

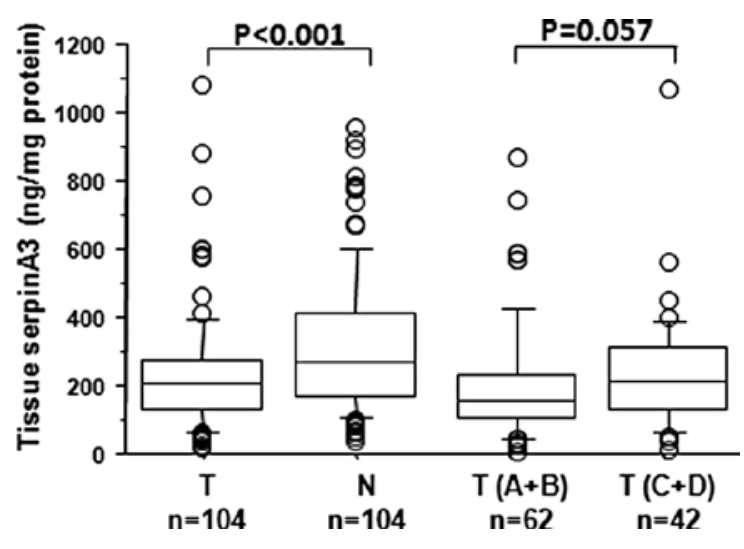

Figure 1. Protein levels of serpinA3 in colorectal tissue from 104 colorectal cancer patients. The levels of serpinA3 in tumour tissue $(\mathrm{T})$ were significantly lower compared to normal $(\mathrm{N})$ paired tissue. The serpinA3 levels in tumour tissues were suppressed in localized disease (Dukes' $\mathrm{A}+\mathrm{B}$ ) in relation to disseminated disease (Dukes' C+D).

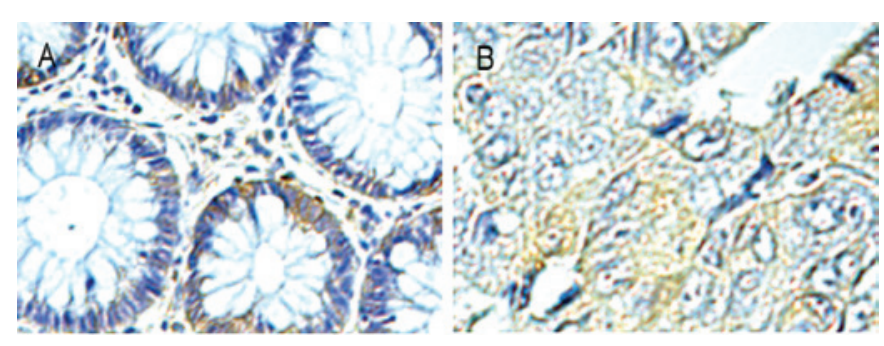

Figure 2. Immunohistochemical detection and localization of serpinA3 in colorectal tissue from colorectal cancer patients. Detection of serpinA3 in (A) normal and (B) cancerous tissue. Magnification, x200.

with the level in disseminated disease (data not shown). No association was found with other clinical characteristics such as age, gender and tumour location (data not shown).

Plasma levels of serpinA 3 and C-reactive protein. In order to search for a tumour marker and the state of inflammation, the plasma levels of serpinA3 and CRP were measured using ELISA in 133 CRC patients and 122 controls. No significant difference $(\mathrm{P}=0.129)$ was found in the levels of plasma serpinA3 in the patients (median $148 \mu \mathrm{g} / \mathrm{ml}$; range 50-690) in comparison with the controls (median $160 \mu \mathrm{g} / \mathrm{ml}$; range 100-345). Plasma serpinA3 concentrations from CRC patients were not related to age, gender, tumour location or Dukes' stage. In agreement with another study (15), a higher concentration of plasma CRP (median $3 \mu \mathrm{g} / \mathrm{ml}$; range 0-136) was found in the CRC patients in comparison with the controls (median $1 \mu \mathrm{g} / \mathrm{ml}$; range 0-27), with a statistical difference between these groups $(\mathrm{P}<0.001)$. Moreover, plasma serpinA3 in the CRC patients showed a positive correlation with $\mathrm{CRP}(\mathrm{r}=0.54, \mathrm{P}<0.001)$ and in the controls $(\mathrm{r}=0.30, \mathrm{P}<0.01)$ (data not shown).

Location of serpinA3 expression in colorectal cancer tissue. Immunohistochemistry was performed to confirm and detect localisation of serpin A3 expression. Focal immunoreactivity from absent to detectable was noted in epithelial cells of the cancer and normal tissue (Fig. 2). A heterogeneous extracellular distribution of serpin A3 was also observed within bands 
Table I. Genotypic and allelic distribution expressed as the percentage and frequency of the serpinA3 gene polymorphism (rs4934) in CRC patients and controls.

\begin{tabular}{|c|c|c|c|c|c|}
\hline & \multicolumn{3}{|c|}{ Genotype $\mathrm{G} \rightarrow \mathrm{A}, \%(\mathrm{n})^{\mathrm{a}}$} & \multicolumn{2}{|c|}{ Allele, $\%(\mathrm{n})^{\mathrm{b}}$} \\
\hline & $\mathrm{G} / \mathrm{G}$ & G/A & $\mathrm{A} / \mathrm{A}$ & G & A \\
\hline $\mathrm{CRC}$ & $25.7(80)$ & $50.2(156)$ & $24.1(75)$ & $50.8(316)$ & $49.2(306)$ \\
\hline Controls & $23.1(83)$ & 52.7 (189) & $24.2(87)$ & $49.4(355)$ & $50.6(363)$ \\
\hline
\end{tabular}

CRC patients vs. controls, not statistically significant. ${ }^{a}$ Total $n=311$ CRC patients and 359 controls; ${ }^{\text {btotal }} \mathrm{n}=622 \mathrm{CRC}$ patients and 718 controls.

Table II. Genotype and allelic frequency of the serpinA3 gene polymorphism (rs4934) in regards to the clinicopathological characteristics of the CRC patients.

\begin{tabular}{lccccc}
\hline & \multicolumn{3}{c}{ Genotype } & & \multicolumn{2}{c}{ Allele } \\
\cline { 2 - 4 } & G/G & G/A & A/A & G & A \\
\hline Tumour site & 38 & 75 & 36 & 151 & 147 \\
Rectum (n=149) & 42 & 81 & 39 & 165 & 159 \\
Colon (n=162) & & & & 182 & 180 \\
Duke's stage & 46 & 90 & 45 & 134 & 126 \\
A+B (n=181) & 34 & 66 & 30 & & \\
C+D (n=130) & & & & & \\
\hline
\end{tabular}

Colon vs. rectum and Dukes' A+B vs. Dukes' C+D, not statistically significant.

of stroma and in some stromal cells with the morphological characteristics of leukocytes. No staining was observed with the isotypic IgG antibody which was used as a negative control (data not shown).

SerpinA3 genotype. To analyse the effect of the serpinA3 gene polymorphism on colorectal carcinogenesis a Taq Man system was used to ascertain the allelic and genotype frequencies in the CRC patients and a control group. No significant difference in genotype distribution was noted between the CRC patients and control subjects or in allelic frequencies (Table I).

Significant differences were not observed following subdivision of the patients into groups according to rectal and colonic cancer or localised (Dukes' $\mathrm{A}+\mathrm{B}$ ) and disseminated (Dukes' $\mathrm{C}+\mathrm{D}$ ) disease (Table II). When assessing the levels of serpinA3 protein in the analysed tissue and plasma samples or subdividing the patients into groups according to clinicopathological characteristics, we were unable to identify any significant differences in genotype or in allelic frequencies (data not shown). Neither the patient nor the control group showed significant deviation from the Hardy-Weinberg equilibrium.

Cancer cell invasiveness. To evaluate the effect of serpin A3 on the invasiveness of Caco-2 and HT-29 cells, serpinA3 was added to the cell suspensions at various concentrations $(0,10,100$ and $2,000 \mathrm{ng} / \mathrm{ml})$. Neither of the cell lines exposed to serpinA3 for 22 or $44 \mathrm{~h}$ exhibited a significant difference in invasiveness through the Matrigel basement membrane compared with that of the unexposed controls.

\section{Discussion}

It has been widely reported that proteinases that are expressed in cancerous tissue promote invasiveness and tumour progression (1-5). Recently, it has been reported that cathepsin G activates pro-MMP-9 (16) and that negative MMP-9 expression is associated with a longer survival time in CRC patients (17).

Observations suggest that serine protease inhibitors exert inhibitory activity against proteases in the control of events associated with inflammatory reactions and connective tissue turnover $(6,7)$. Recently it has been reported that serpinA3 is aberrantly expressed in metastatic melanoma (18).

The expression profile of serpinA3 in human CRC has rarely been studied. Immunohistochemistry was used to identify the cellular source and localisation of serpinA3 protein in CRC patients, and immunoreactivity was noted in the compartment of the cancer and normal tissue and also in the stroma and stromal cells such as leukocytes and fibroblasts. Moreover, CRC patients assessed using ELISA were shown to have a significantly lower level of serpinA3 protein in cancer tissues in comparison with paired normal tissues. The protein level of serpinA3 was found to be significantly positively correlated in the cancerous and normal tissue. This indicates that there may be some basic individual differences. To assess 
the results according to disease severity the patients were divided according to localised (Dukes' $\mathrm{A}+\mathrm{B}$ ) and disseminated (Dukes' $\mathrm{C}+\mathrm{D}$ ) disease. We found that serpinA3 expression was higher in disseminated disease.

Based on the balance between its proteolytic and inhibitory activity in tissue and our results that serpinA 3 expression in cancer tissue is lower than that in paired normal tissues it may be suggested that a high activity of proteinases is linked to tumour growth and aggressiveness. Proteinases play a role in cancer metastasis (1-5). Notably, the down-regulated serpin A3 expression in cancer tissue noted in the present study appears to depend on regulatory factors secreted from tumour cells. However, our results appear in part to be inconsistent due to the fact that a higher serpinA3 concentration was associated with malignant potency such as disseminated (Dukes' $\mathrm{C}+\mathrm{D}$ ) disease. Further investigation is required to explain this discrepancy. Notably, serpinA3 uses conformational change, a change-based trapping mechanism, to inhibit target enzymes (6). Moreover, serpinA3 is an inflammatory protein and is regulated by cytokines among which the inflammatory cytokine interleukin-6 (IL-6) is the major regulator (19). This cytokine is also elevated in CRC (20). Considering the relationship between cancer and inflammation, cytokines appear to contribute to tumour growth, but also to effective antitumour immunity $(21,22)$. Studies related to structure-function relationships, the regulatory pathway for serpin A3 expression in CRC and the method by which serpinA3 affects invasion and metastasis in tumour cells are warranted. However, in the present study, we found that cells derived from CRC, such as Caco-2 and HT-29, did not reveal any change in invasiveness following incubation with serpin A3 in vitro. Thus, other mechanisms may be involved in vivo.

Studies indicate that the tissue expression of serpinA3 in gastric cancer (10) and melanoma (18) is correlated with a higher stage of disease and is inversely correlated with overall survival. In a forthcoming study, it may be informative to investigate the manner in which tissue levels of serpin A3 affect the survival rate of CRC patients.

To investigate serpin $\mathrm{A} 3$ as a potential tumour marker and the state of inflammation, the plasma levels of serpinA3 and CRP were measured. No significant difference was noted in the levels of plasma serpinA3 in patients in comparison to the controls and no associations were found between these levels and a series of clinical characteristics. The functional consequence of this relationship may disqualify circulating serpinA3 as a tumour marker with impact on CRC. In agreement with another study (15), a higher concentration of plasma CRP was found in CRC patients compared to the controls. Moreover, plasma serpin A3 and CRP of the CRC patients and controls were significantly positively correlated. SerpinA3 concentrations in plasma appear to be correlated with systemic inflammatory response expressed by the inflammatory protein CRP, which appears not to be specific to CRC patients. On the other hand, it has been questioned whether circulating levels of CRP are a useful indicator of CRC (23).

No studies have been carried out in CRC patients to elucidate the consequences of the common SNP variant (rs4934) in the serpinA3 signal sequence gene. We aimed to determine whether an association exists between this gene variant and CRC. The genotype distributions and allelic frequencies for the gene variant were not significantly associated with CRC. Furthermore, we investigated whether this SNP is a potential candidate affecting the expression of serpinA3. However, we were unable to detect any association between the genotype and plasma or tissue concentration of serpinA3.

Overall, the present study aimed to assess the significance of serpinA3 levels in CRC patients. SerpinA3 was found to be down-regulated in cancerous tissue as compared to paired normal tissue, while this suppression was lower at a severe disease stage. Moreover, in vitro, exogenous serpinA3 had no impact on the invasiveness of CRC cells.

Further studies are warranted to improve our understanding of the role of serpinA3 in CRC. The data presented in this study are prerequisite to a forthcoming study on progression and the 5-year survival rate in CRC.

\section{Acknowledgements}

This study was supported by grants from Futurum The Academy of Healthcare, County Council of Jönköping, Sweden, the Foundation of Clinical Cancer Research, Jönköping, Sweden and the University College of Health Sciences, Jönköping Sweden.

\section{References}

1. Lopez-Otin C and Matrisian LM: Emerging roles of proteases in tumour suppression. Nat Rev Cancer 7: 800-808, 2007.

2. Stetler-Stevenson WG: The role of matrix metalloproteinases in tumor invasion, metastasis and angiogenesis. Surg Oncol Clin N Am 10: 383-392, 2001.

3. Murray GI, Duncan ME, O'Neil P, Melvin WT and Fothergill JE: Matrix metalloproteinase-1 is associated with poor prognosis in colorectal cancer. Nat Med 2: 461-462, 1996.

4. Nikkola J, Vihinen P, Vlaykova T, Hahka-Kemppinen M, Kahari VM and Pyrhonen S: High expression levels of collagenase-1 and stromelysin-1 correlate with shorter diseasefree survival in human metastatic melanoma. Int J Cancer 97: 432-438, 2002.

5. Kuester D, Lippert H, Roessner A and Krueger S: The cathepsin family and their role in colorectal cancer. Pathol Res Pract 204: 491-500, 2008.

6. Law RHP, Zhang Q, McGowan S, Buckle AM, Silverman GA, Wong W, Rosado CJ, Langendorf CG, Pike RN, Bird PI and Whisstock JC: An overview of the serpin superfamily. Genome Biol 7: 216, 2006.

7. Gettins PG: Keeping the serpin machine running smoothly. Genome Res 10: 1833-1835, 2000.

8. Kalsheker NA: Alpha-1-antichymotrypsin. Int J Biochem Cell Biol 28: 961-964, 1996.

9. Janciauskiene $\mathrm{S}$ : Conformational properties of serine proteinase inhibitors (serpins) confer multiple pathophysiological roles. Biochim Biophys Acta 1535: 221-235, 2001.

10. Allgayer H, Babic R, Grutzner KU, Beyer BCM, Tarabichi A, Schildberg FW and Heiss MM: Tumor-associated proteases and inhibitors in gastric cancer: analysis of prognostic impact and individual risk protease patterns. Clin Exp Metastasis 16: 62-73, 1998.

11. Kamboh ML, Minster RL, Kenney M, Ozturk A, Desai PP, Kammerer CM and DeKosky ST: Alpha-1-antichymotrypsin (ACT or SERPINA3) polymorphism may affect age-at-onset and disease duration of Alzheimer's disease. Neurobiol Aging 27: 1435-1439, 2006.

12. Slowik A, Borratynska A, Turaj W, Pera JP, Dziedzic T, Figlewicz DA, Betlej M, Krzyszkowski T, Czepko R and Szczudlik A: Alpha-1-antichymotrypsin gene (SERPINA3) A/T polymorphism as a risk factor for aneurysmal subarachnoid hemorrhage. Stroke 36: 737-740, 2005.

13. Liu W, Zhu Y, Ge M, Pang Q and Yu Y: Polymorphism rs4934 of SERPINA3 and sporadic intracranial aneurysms in the Chinese population. Cerebrovasc Dis 29: 68-72, 2010. 
14. Krischek B, Akagawa H, Tajima A, Narita A, Kasuya H, Hori $\mathrm{T}$ and Inoue I: The alanine/threonine polymorphism of the alpha-1-antichymotrypsin (SERPINA3) gene and ruptured intracranial aneurysms in the Japanese population. Cerebrovasc Dis 23: 46-49, 2007.

15. Erlinger TP, Platz EA, Rifai N and Helzlsouer KJ: C-reactive protein and the risk of incident colorectal cancer. JAMA 291: 585-590, 2004.

16. Wilson TJ, Nannuru KC and Singh RK: Cathepsin G-mediated activation of pro-matrix metalloproteinase 9 at the tumor-bone interface promotes transforming growth factor- $\beta$ signaling and bone destruction. Mol Cancer Res 7: 1224-1233, 2009.

17. Bendardaf, R, Buhmeida A, Hilska M, Laato M, Syrjänen S, Syrjänen K, Collan Y and Pyrhönen S: MMP-9 (gelatinase B) expression is associated with disease-free survival and diseasespecific survival in colorectal cancer patients. Cancer Invest 28: 38-43, 2010.

18. Wang Y, Jiang H, Dai D, Su M, Martinka M, Brasher P, Zhang Y, McLean D, Zhang J, Ip W, Li G, Zhang X and Zhou Y: Alpha 1 antichymotrypsin is aberrantly expressed during melanoma progression and predicts poor survival for patients with metastatic melanoma. Pigment Cell Melanoma Res 23: 575-578, 2010.
19. Castell JV, Gomez-Lechon MJ, David M, Andus T, Geiger T, Trullenque R, Fabra R and Heinrich PC: Interleukin- 6 is the major regulator of acute phase protein synthesis in adult human hepatocytes. FEBS Lett 242: 237-239, 1989.

20. Chung YC and Chang YF: Serum interleukin-6 levels reflect the disease status of colorectal cancer. J Surg Oncol 83: 222-226, 2003.

21. Coussens LM and Werb Z: Inflammation and cancer. Nature 420: 860-867, 2002.

22. Balkwill F: Chemokine biology in cancer. Semin Immunol 15: 49-55, 2003.

23. Trichopoulos D, Psaltopoulou T, Orfanos P, Trichopoulou A and Boffetta P: Plasma C-reactive protein and risk of cancer: a prospective study from Greece. Cancer Epidemiol Biomarkers Prev 15: 381-384, 2006. 